\title{
Study of Au/Pd and Au/Co Bimetallic Nanoparticles Using Aberration Corrected STEM
}

Nabraj Bhattarai, Subarna Khanal, Daniel Bahena, Arturo Ponce and Miguel Jose-Yacaman

Department of Physics and Astronomy, University of Texas at San Antonio, One UTSA Circle, San Antonio, TX, 78249

The synergetic combination of two metals in bimetallic (BM) nanoparticle (NP) significantly changes the optical, catalytic, electronic and magnetic properties. The possible reason for synergetic effects is the heterometallic bond between two metals, dilution of active site, the combination of two kinds of metals and their fine structures. The enhanced catalytic properties of BM nanoparticles are widely used in several industrial reactions. For practical applications and better understanding of this system, there should be well-controlled synthesis of shape and size selected structures, powerful structural characterization tools including advanced in situ atomic-level analytical techniques, modern modeling tools with supercomputers, and in-depth understanding of structure-property relationship. In this paper, we are presenting the use of Z-contrast imaging in aberration-corrected HAADF STEM images in the study of atomic ordering in nanoalloys and core/shell and also investigating the growth mechanism in $\mathrm{Au}-\mathrm{Pd}$ and the interface region between $\mathrm{Au}$ and $\mathrm{Pd}$.

The strain produced from lattice mismatch in Au-Pd is released through the strain release mechanism [1]. An example is presented in left frame of figure 1, which shows that first Shockley partial dislocations (SPD) combined with stacking faults (SF) appear at the last Pd layer; then, as the shell grows the SPDs and SFs appear at the interface and combine with misfit dislocations, which finally diffuse to the free surfaces due to the alloying of Au into the Pd shell. The critical layer thickness was found to be at least $50 \%$ greater than in thin films, confirming that shell growth on nanoparticles can sustain more strain due to the tridimensional nature of the nanoparticles. The growth mechanism and the shape evolution of $\mathrm{Au}-\mathrm{Pd}$ core-shell nanoparticles from single crystal seed to final concave nanocube presented in right frame of figure 1 [2]. The study showed that the concave nanocubes grew from octahedral Au seeds due to fast growth along $\langle 111\rangle$ directions and presented high index facet (HIF) surfaces that will increase the catalytic activity of different reactions.

The chemical ordering in $\mathrm{Au} / \mathrm{Co}$ magnetoplasmonic ferromagnetic nanoparticle is studied using aberration corrected HAADF STEM images and is presented in figure 2. The decahedral shaped $\mathrm{Au} / \mathrm{Co}$ NPs along [110] zone axis with different intensities can be seen. The intensity profile is measured along the direction shown and is presented in the lower frame of figure 2 . The stronger intensity region corresponds to Au-rich atomic columns while the weaker intensity region corresponds to Co-rich atomic columns. The HAADF STEM image result is verified using energy dispersive X-ray spectroscopy (EDS) and also Grand Canonical Monte Carlo simulations [3].

\section{References:}

[1] N. Bhattarai, G. Casillas, A. Ponce and M. Jose-Yacaman, Surface Science, 2013, 609, 161-166.

[2] N. Bhattarai, G. Casillas, S. Khanal, J. J. Salazar, A. Ponce and M. Jose-Yacaman, J Nanopart Res, 2013, 15, 1-13.

[3] N. Bhattarai, G. Casillas, S. Khanal, D. Bahena, J. J. Velazquez-Salazar, S. Mejia, A. Ponce, V. P. Dravid, R. L. 
Whetten and M.M. Mariscal, MRS Communications, 2013, 3, 177-183.

[4] The author would like to acknowledge following grants: NCRS (5 G12RR013646-12), National Institute on Minority Health and Health Disparities (G12MD007591), NSF DMR-1103730, NSF PREM Grant \# DMR 0934218; Welch Foundation (grant No. AX-1615).
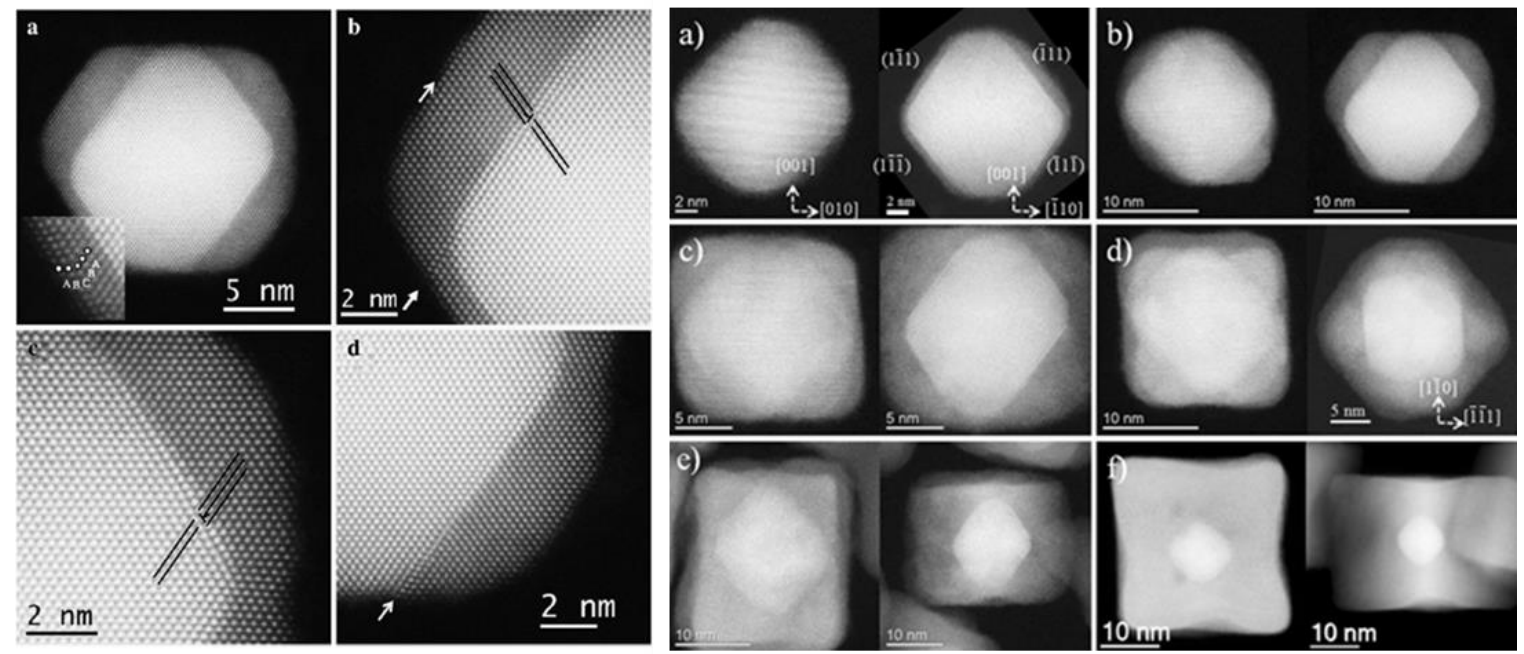

Figure 1: HAADF STEM images of Au-Pd core-shell nanoparticles with varying Pd thickness. Left: stacking fault and partial dislocation in Au-Pd core-shell nanoparticles, Au (core) and Pd (shell). Right: Au-Pd core-shell nanoparticles with different Pd thickness where the growth process can be observed. Frames on the left present nanoparticles in a [100] zone axis, while on the right present nanoparticles in a [110] zone axis, except the right frame in d) which is a [112] zone axis. The zone axis is inserted in the corresponding images and the crystallographic orientation is also inserted.
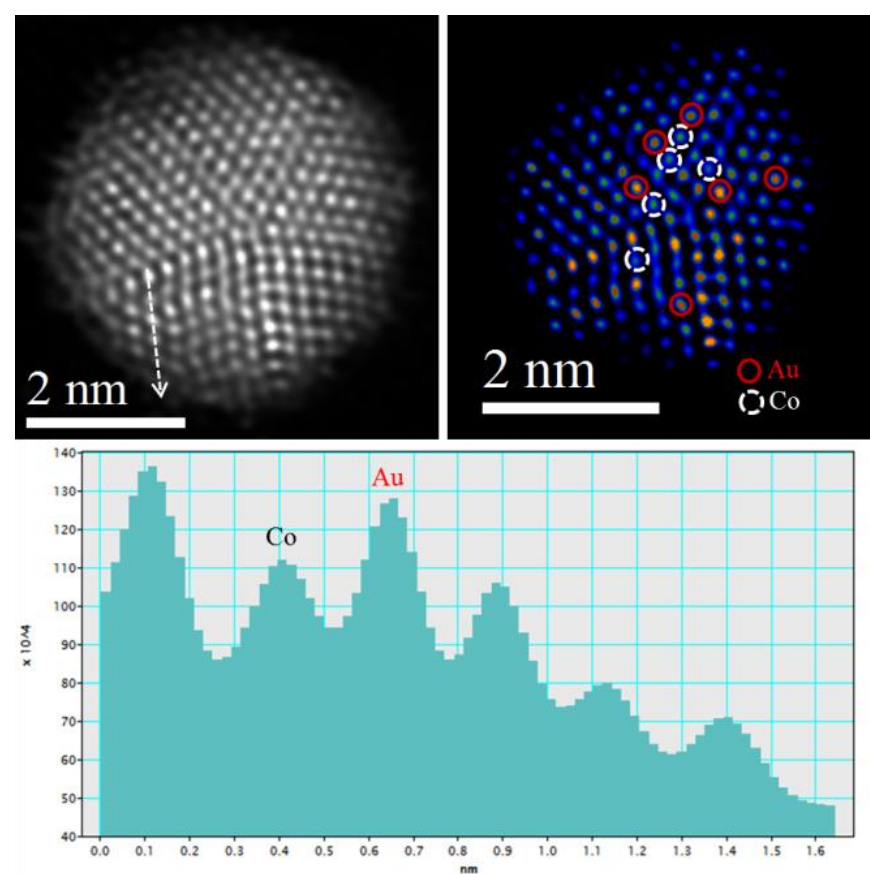

Figure 2: HAADF STEM image of Au/Co decahedral nanostructure with different intensity for atomic columns: the higher intensity for $\mathrm{Au}$ rich atomic columns and lower intensity for Co rich atomic columns. A line profile shows the variation in intensities along that direction. 